\title{
Circuit
}

Musiques contemporaines

\section{Cage en liberté : réflexions libres}

\section{Maxime McKinley}

Volume 13, numéro 3, 2003

Électroacoustique : nouvelles utopies

URI : https://id.erudit.org/iderudit/902290ar

DOI : https://doi.org/10.7202/902290ar

Aller au sommaire du numéro

Éditeur(s)

Les Presses de l'Université de Montréal

ISSN

1183-1693 (imprimé)

1488-9692 (numérique)

Découvrir la revue

Citer ce compte rendu

McKinley, M. (2003). Compte rendu de [Cage en liberté : réflexions libres]. Circuit, 13(3), 103-108. https://doi.org/10.7202/902290ar

Ce document est protégé par la loi sur le droit d'auteur. L'utilisation des services d'Érudit (y compris la reproduction) est assujettie à sa politique d'utilisation que vous pouvez consulter en ligne.

https://apropos.erudit.org/fr/usagers/politique-dutilisation/
Cet article est diffusé et préservé par Érudit.

Érudit est un consortium interuniversitaire sans but lucratif composé de l'Université de Montréal, l'Université Laval et l'Université du Québec à Montréal. Il a pour mission la promotion et la valorisation de la recherche. https://www.erudit.org/fr/ 


\title{
Cage en liberté : réflexions libres
}

\author{
Maxime McKinley
}

L'événement Cage en liberté, présenté à la salle Pierre-Mercure, le 14 mai 2002, par l'Ensemble Contemporain de Montréal (ECM), a remporté cette année deux prix majeurs : d'abord le prix Opus "événement de l'année", puis celui du Conseil des arts de Montréal. Un concert d'une telle importance, d'un tel succès, mérite un retour, une réflexion. Soulignons l'ironie du sort: Cage, qui passa sa vie à désacraliser l'art, est finalement devenu une vache sacrée, à laquelle on fait des hommages, hommages à leurs tours couverts d'hommages, le tout se retrouvant sujet de réflexion dans une revue de musique contemporaine! Bref, le paradoxe de l'« anartiste » devenu un "phare »...

Je joverai donc le jeu ici, d'abord en proposant un témoignage assez détaillé de l'événement Cage en liberté. Ensuite, à la manière d'une postface, je me ferai un devoir de pousser la réflexion plus loin. Cage a légué une proposition esthétique d'une force exceptionnelle : quelle résonance a-telle aujourd'hui ? Enfin, toujours en guise de postface, je tenterai de cerner l'approche stratégique et artistique de l'ECM, qui semble de plus en plus miser sur l'événementiel pour transmettre la musique d'aujourd'hui.

\section{Cage en liberté : témoignage d'une réception}

C'est pour souligner le $10^{\mathrm{e}}$ anniversaire de la mort de Cage que l'ECM organisait ledit événement, composé de trois concerts et de trois MusiCircus lexpositions mobiles d'installations et de performances, sonores ou visuelles, qui étaient réparties sur les trois étages de la salle de concert Pierre-Mercure). Le tout avait une durée d'environ quatre heures et y figurait une grande quantité d'artistes et de projets. Lors des concerts ont été entendues trois créations en hommage à Cage (Lesage, Beauchesne, Villeneuve) et différentes œuvres de Cage lui-même. Chacun des concerts était organisé autour d'une période précise de la production de Cage lécriture, hasard, videl, tandis que les MusiCircus agissaient comme des intermèdes surréalistes et dadaïstes. 


\section{MusiCircus}

Les MusiCircus étaient exactement calqués sur le type de happening que créait Cage. II s'agit d'un univers riche en contenu latent, qui implique une désacralisation de l'art, une quête de la non-intention détruisant le " Je " romantique, une approche totalement inclusive qui ne fait aucune discrimination, un processus de création axé non pas sur la mémoire et la durabilité mais sur le seul instant présent. Cela se manifestait par l'emploi de matériaux tirés du quotidien (anti-art), par la simultanéité de toutes ces installations rendant relativement anonyme chacune d'entre elles et par une complexité ne pouvant être due qu'à une importante dimension aléatoire rendant impossible toute reproduction ultérieure.

Je ne donnerai ici que quelques exemples d'installations et de performances. Dans le domaine de l'art visuel, le peintre Katsumi Kimoto peignait en direct trois tableaux en employant diverses techniques propres aux américains contemporains de Cage. Une performance de Dominique Porte, accompagnée de Sara Hanley, nous amenait dans l'univers de la danse, rappelant la longue collaboration de Cage avec le chorégraphe Merce Cunnigham. Mentionnons enfin Marc Hyland et Silvio Palmieri, qui faisaient jouer simultanément diverses musiques, ou encore les improvisations d'esprit bruitiste de Jean Derôme.

II se dégageait de ces MusiCircus une sorte d'esprit festif, dégageant lui-même une électricité extrêmement prégnante. En somme, un foisonnement grouillant sans cesse, car en plus des nombreuses installations, il y avait toute la circulation, les embouteillages et le bruit de fond du public.

\section{Concerts}

Le premier concert s'ouvrait avec la création du Quatuor à cordes /I de Jean Lesage, interprété par le quatuor Bozzini. L'œuvre était fidèle à la manière de lesage : hautement raffinée, ambiguë, d'un humour distant. Ici, les liens à faire avec Cage sont de l'ordre de la dialectique, car Lesage ne semble pas avoir fait plus "Cage " que $d^{\prime}$ habitude. Du reste, ce que suggère comme possibilités de réflexions une dialectique entre la pensée de Cage et celle de lesage laxée sur la postmodernité et le baroquel est riche et fait constater des similitudes. Par exemple, la notion de "second degré ": qu'il s'agisse d'une citation chez lesage ou d'un espace sonore new-yorkais chez Cage, procède de la même démarche sémiologique de « recontextualisation". Suivaient les Sonates et Interludes pour piano préparé, de John Cage, que Brigitte Poulin nous a fait entendre dans une interprétation extrêmement 
poétique et raffinée, alternant jeu éthéré et jeu plus mordant, alternance d'ailleurs latente dans l'œuvre même : l'aspect bruité, impur, voire "parasite " du piano préparé s'inscrit dans une œuvre à l'écriture dépouillée et au caractère onirique.

Le second concert était, à mon sens, la clé de voûte de l'événement. Le concept du compositeur Alain Beauchesne, était d'habiller sa pièce L'heure de s'enivrer de diverses œuvres de Cage. Ces éléments étaient superposés pour la première fois lors du concert, aucune entente n'avait été prise au préalable à l'exception de la durée d'exécution et ainsi, le résultat s'est situé, comme le dit Beauchesne, "quelque part entre un minimum et un maximum d'activité sonore, soit le silence et la cacophonie". Je trouve que par ce concept, l'hommage de Beauchesne était une fort belle réussite, dans la mesure où il y avait non pas éclipse entre l'honorant et l'honoré, mais rencontre. L'œuvre de Beauchesne, quasi stravinskienne, semble d'ailleurs se situer à un tournant dans la production du compositeur.

La pièce de Villeneuve, Harmonia-Geometria-Nostalgia, qui ouvrait le troisième et dernier concert, était pleine d'un lyrisme ambigu, harmoniquement très mystérieuse, évoquant un univers tonal sans l'être vraiment. Je crois que Villeneuve, tout comme Lesage, aurait pu écrire la même œuvre dans un contexte non cagien. Cela dit, on y sentait un certain dépouillement pouvant rappeler Cage. Considérant qu'il s'agit d'un hommage à ce dernier, j'ai trouvé très intéressante la gravité de la pièce qui jetait loin la rigolade! Ce concert se terminait avec une œuvre soulignant l'orientalisme chez Cage, dans une poétique zen du vide (du silence) et du détachement : Fourteen, pour piano à archet et ensemble, de Cage lui-même. C'est une œuvre d'une extrême beauté, chacun des musiciens faisant des sons tenus, générant un statisme fascinant en même temps qu'une densité en constante transformation. Le son du piano à archet (ce dernier consistant en une corde de nylon) est une pure merveille: c'est un timbre d'un onirisme et d'une douceur incomparables. Enfin, l'œuvre était visuellement très belle : l'ensemble bougeant à peine, le soliste se déplaçant autour du piano avec son archet et le chef offrant comme seule métrique une sorte de mouvement circulaire des bras, rappelant celui des aiguilles d'une horloge.

\section{Cage et les débats esthétiques d'aujourd'hui}

Le fait de consacrer un événement d'une telle importance à Cage, et que cela soit un réel succès, pose la question de l'actualité du compositeur: de sa postérité. En ce sens, je crois qu'il peut être intéressant de se questionner sur les liens entre l'univers conceptuel de Cage et les principaux débats esthétiques d'aujourd'hui.

Prenons, par exemple, la dialectique de la modernité/postmodernité. Cage, en "contextualisant" en œuvres d'art des univers sonores tirés du quotidien (ready- 
madel, s'attaquait en fait à toute forme de système de valeurs entourant le phénomène de l'art. C'était là sa table rase dadaïste, moderne : l'abolition de toute forme de critères de jugements de valeurs, une totalisation de l'art. De là naîtra son conflit avec Boulez, ce dernier ayant toujours conservé une certaine grille de jugement relative à la qualité d'une œuvre. En ce sens, on peut voir Cage comme étant plus radicalement moderne que Boulez. En revanche, la postmodernité a beaucoup critiqué le "purisme" moderne, l'approche exclusive plutôt qu'inclusive. En ce sens, Cage est souvent perçu comme un outsider du dogmatisme moderne et parfois comme un précurseur de la postmodernité. Ainsi, dans un contexte où l'on peut se demander si l'antithèse moderne/postmoderne ne s'essouffle pas un peu, si le besoin d'une certaine synthèse, d'une voie de sortie, n'est pas palpable, le cas de Cage a certainement de quoi stimuler encore aujourd'hui !

Depuis quelque temps est particulièrement omniprésent un questionnement concernant la fonction sociale de l'art. Les avant-gardes du xx siècle s'étant beaucoup appliqué à faire tomber toute forme de convention dans l'art, notamment au nom d'idéaux de nouveauté et de liberté, la communication avec le public a bien sûr été beaucoup plus difficile. Parallèlement à cela, la montée des mass-médias et de l'industrie culturelle a suivi la courbe inverse : rendre toute forme d'expression le plus facile possible à assimiler, au risque de tomber dans l'insignifiance. Tout ceci pose évidemment de grandes questions...

En musique par exemple, la place du concert de musique dite savante est, depuis quelques années, fortement ébranlée. Face à l'incroyable efficacité de la technologie audio, que reste-t-il du concert? Que peuvent être, aujourd'hui, les fonctions sociales d'un concert de musique dite savante? À l'instar de la musique pop, la musique dite savante devraitelle amplifier la dimension événementielle intrinsèque de chaque concert? Mais n'est-ce pas là risquer de tomber dans les lois de l'offre et de la demande, de perdre une certaine forme de liberté intellectuelle de tout temps fondamentale à l'être humain ? La démarche de Cage, par rapport à ces questions, est très intéressante. Dans un monde de plus en plus atomisé, de plus en plus individualisé, il arrivait, avec une profonde authenticité artistique, par ses happenings, à rassembler un vaste public dans un seul lieu et, à des lieves de l'insignifiance, à créer de véritables momentum collectifs. Question fonction sociale de l'art, de la musique, n'est-ce pas, aujourd'hui encore, très honorable et même inspirant?

\section{L'ECM et le renouvellement du concert}

Je crois qu'avec Cage en liberté, l'ECM a su démontrer l'apport considérable, pour le concert et son public, d'une forte dimension événementielle. La SMCQ a maintes fois eu recours à ce must dans ses débuts et, de toute évidence, elle y 
retourne de nouveau : mentionnons la Symphonie du millénaire ou, récemment, le premier festival Montréal/Nouvelles Musiques. Le Forum du NEM est, aussi, toujours très stimulant.

Un exemple de stratégie employée par l'ECM et qui déclenche cette ferveur artistique tout en réussissant à attirer un large public, c'est la dimension pluridisciplinaire de leurs événements. Ce fut le cas avec Cage en liberté tout comme les concerts Unions libres, où un compositeur est jumelé à un artiste d'une autre discipline, afin de réaliser un projet conjoint. Cette approche, d'une grande efficacité, rejoint un plus vaste public et prouve qu'une grande réflexion d'ordre sociologique a été faite.

La musique, comme l'art en général, n'a-telle pas une fonction d'échange, de rassemblement? Tout comme la peinture a dû, face à la photographie, raffermir ses fondements mêmes, le concert ne doitil pas se redéfinir, se poser comme autonome par rapport aux mass-médias? Dans cette direction, Cage est une mine d'or d'inspiration...

\section{Ouverture : Cage plus actuel que jamais?}

Au moment de l'événement Cage en liberté, John Cage était mort depuis dix ans. Cependant, je crois que son héritage peut encore agir comme une fontaine de Jouvence, même si nos préoccupations actuelles n'ont pas nécessairement été les siennes. L'art, en plein changement de siècle, en plein changement de millénaire, traverse une crise ou, du moins, éprouve du mal à se définir. Peut-être est-ce un peu la faute lou l'apport!) de gens comme John Cage, à l'époque de la "mort de l'art »? Lorsqu'une prise de son des rues de New York a le même statut d'œuvre qu'une fugue de Bach, que reste-til des critères de définitions de l'art? On pouvait reconnaître Cage de son vivant comme un grand "anartiste" à la Duchamp, un grand "déconstructeur". Mais, paradoxalement, l'œuvre et la pensée de Cage, face à toutes les questions que pose aujourd'hui l'esthétique, se révèlent extrêmement riches de stimulations, de réflexions, voire de pistes de solutions. Ainsi, avec la richesse de son héritage générant des événements comme Cage en liberté, on ne peut que parler d'un être profondément fertile et fertilisant, d'un constructeur : bref, d'un artiste. Ainsi va l'histoire... 


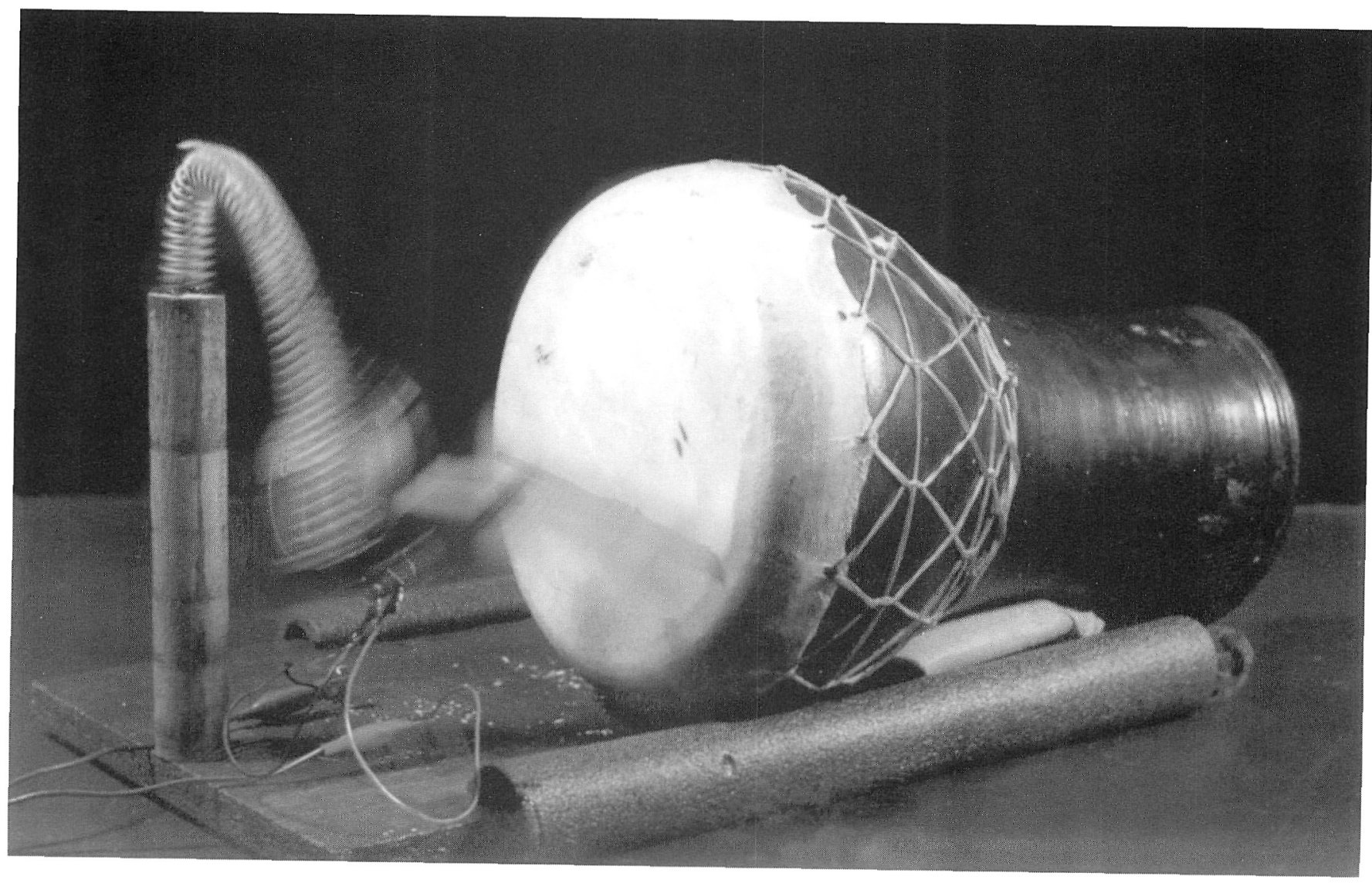

